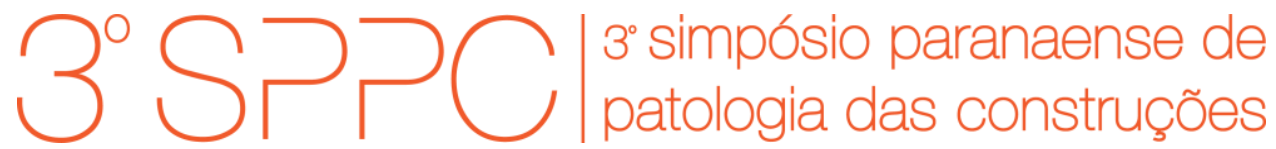

ISSN 2526-7248 artigo n. 3SPPC1015, pp. 166-173, 2018

\title{
Aplicação de modelo para estimar a umidade relativa em ambiente marinho
}

\author{
Medeiros-Junior, Ronaldo A. ${ }^{1}$; Müller, Rodrigo Oliveira ${ }^{2}$ \\ 1Professor, Universidade Federal do Paraná, medeirosjunior.ufpr@gmail.com \\ ²Engenheiro civil, Universidade Tuiuti do Paraná, eng.rodrigomuller@gmail.com
}

Resumo: A umidade relativa do ambiente é um fator importante no desenvolvimento de vários processos de degradação do concreto. Essa variável climática é muitas vezes requisitada em modelos de durabilidade das estruturas. Assim, em manifestações patológicas como corrosão de armadura, reação álcali-agregado, carbonatação, dentre outras, o entendimento deste fator climático é de extrema necessidade. Contudo, a falta da medição deste parâmetro por estações meteorológicas pode dificultar a aplicação de modelos de durabilidade para uma determinada região. Neste sentido, modelos são disponíveis na literatura para estimar a umidade relativa a partir de outras variáveis climáticas e suprir esta lacuna de dados. Este artigo tem o objetivo de avaliar a aplicação de um modelo para estimar a umidade relativa em ambiente marinho. Os dados de umidade relativa estimados através de modelo matemático foram comparados com dados meteorológicos obtidos pelo Instituto Nacional de Meteorologia em um período de 30 anos. Foi verificada proximidade entre os valores estimados e medidos, com diferença percentual inferior a $10 \%$. Também foi observado que o modelo tende a superestimar os valores de umidade relativa para 0 ambiente marinho. Análises estatísticas foram realizadas para verificar os dados. $O$ coeficiente de correlação $(r)$ e o índice de concordância $(d)$ foram 0,71 e 0,62, respectivamente, representando uma condição satisfatória para aplicação do modelo.

Palavras-chave: umidade relativa, modelo, durabilidade, ambiente marinho.

Abstract: Relative humidity of the environment is an important factor in the development of various degradation processes. This climatic variable is often required in durability models of concrete structures. Thus, in pathological manifestations such as reinforcement corrosion, alkali-aggregate reaction and carbonation, this climatic factor is extremely necessary. However, failure to measure this parameter by meteorological stations may make it difficult to apply durability models for a given region. In this sense, models are available in the literature to estimate relative humidity from other climatic variables and to fill this data gap. This paper aims to evaluate the behavior of a model to estimate the relative humidity in the marine environment. The relative humidity data estimated by means of a mathematical model were compared with meteorological data obtained by the National Institute of Meteorology in a period of 30 years. The results show that the values estimated and measured are similar, with percentage difference less than $10 \%$. It was also observed that the model tends to overestimate the values of relative humidity for the marine environment. Statistical analyzes were performed to verify the data. The correlation coefficient $(r)$ and the concordance index $(d)$ were 0.71 and 0.62 , respectively, representing a satisfactory condition for model application.

Keywords: relative humidity, model, durability, marine environment. 
MEDEIROS-JUNIOR, R. A.; MÜLLER, R. O., APLICAÇÃO DE MODELO PARA ESTIMAR A UMIDADE RELATIVA EM AMBIENTE MARINHO. $3^{\circ}$ Simpósio Paranaense de Patologia das Construções (30 SPPC), artigo 3SPPC1015, pp. 166 - 173, 2018. DOI:

\section{Introdução}

O uso de variáveis climáticas é muitas vezes exigido para aplicação de modelos de durabilidade de estruturas de concreto [1-3]. Contudo, a falta de dados contínuos provenientes de estações meteorológicas é um problema persistente que atinge alguns países, como o Brasil, dificultando a aplicação destes modelos de durabilidade. Neste contexto, estimar esses dados é uma alternativa usualmente utilizada para preencher series incompletas [4].

A umidade relativa é um parâmetro fortemente relacionado aos processos de degradação do concreto, como corrosão das armaduras, reação ácali-agregado, carbonatação, dentre outros. A maioria das manifestações patológicas no concreto está relacionada com a presença de água em seu interior, que de certa forma está atrelada com a umidade do meio externo.

Nesse contexto, Castellví et al. [5] propuseram o uso da Equação 1 para estimar a umidade relativa do ambiente.

$$
U R=100 \frac{e_{s}\left(T_{p o}\right)}{e_{S}\left(T_{m}\right)}
$$

Onde $e_{s}\left(T_{p o}\right)$ representa a pressão de saturação de vapor d'água no $\operatorname{ar}(\mathrm{hPa})$, determinada a partir da temperatura do ponto de orvalho $\left({ }^{\circ} \mathrm{C}\right)$, podendo ser substituída pela temperatura mínima (Tn); e $e_{s}\left(T_{m}\right)$ a pressão de vapor d'água de saturação no ar $(\mathrm{hPa})$, calculada a partir da temperatura média horária $\left({ }^{\circ} \mathrm{C}\right)$.

Uma maneira de calcular a pressão de saturação de vapor d'água do ar (es, em $\mathrm{Pa}$ ) é através da Equação 2, proposta por Tetens [6], onde a temperatura (T) é dada em graus Celsius (oC).

$$
e_{S}=610,8 \cdot e^{\left(\frac{17,3 \cdot T}{237,3+T}\right)}
$$

Outra forma de determinar es é por intermédio da Equação 3 dada por Kuo e Raymond [7], e que foi aplicada por Raymond [8] para estudo da advecção da água usando a umidade relativa.

$$
e_{S}=6,11 \cdot \exp \left[25,22\left(1-\frac{273,16}{T}\right)\right] \cdot\left(\frac{273,16}{T}\right)^{5,31}
$$

Dentre os estudos que aplicaram a Eq. 1, Vuille et al. [9] encontraram coerência no uso do método proposto por Castellví et al. [5] para estimativa da umidade relativa, aplicando tal modelo em estudo de observações glaciais das mudanças climáticas na região do tropical dos Andes, no período de 1950 a 1998.

Em geral, observa-se que os modelos de estimativa de umidade relativa são uma ferramenta interessante para locais que possuam poucos dados climatológicos. No entanto, deve-se atentar que diferentes tipos de ambiente externo podem exigir maior precisão de dados de umidade relativa, como é o caso do ambiente marinho.

O meio ambiente marinho pode ser caracterizado como a região que sofre influência do oceano, e a sua área depende das condições das variáveis climáticas do local. A 
urbanização intensa nesse ambiente é responsável pela grande movimentação de pessoas e recursos, principalmente em países em desenvolvimento. Nesse tipo de ambiente, a umidade relativa é um dos parâmetros mais significativos, por exemplo, no que diz respeito à durabilidade de estruturas de concreto [10].

Portanto, este artigo tem por objetivo verificar a precisão do modelo de Castellví et al. [5] para estimar a umidade relativa em ambiente marinho, observando o comportamento do mesmo para algumas cidades da costa brasileira.

\section{Materiais e Métodos}

Os materiais utilizados neste trabalho foram dados de umidade relativa registrados e coletados em estações meteorologicas dispostas ao longo da costa brasileira. $\mathrm{O}$ conjunto de dados ininterruptos de umidade relativa disponíveis para utilização neste estudo abrangeu o período de 1961 a 1990, totalizando 30 anos de dados. Ressalta-se que não foi possível obter, através de estações meteorológicas, uma série de dados contínua para um maior período de tempo ou mais atual do que a mencionada.

De posse dos dados de umidade relativa, o desempenho do modelo de Castellví et al. [5] em estimar a umidade relativa foi avaliado para diferentes cidades no Brasil, localizadas em ambiente marinho. Os critérios para escolha das cidades da costa do Brasil que serviram para análise dos modelos foram:

(1) Cidades localizadas em ambientes que sofrem influência direta do mar, ou seja, cidades litorânea;

(2) Cidades com registros publicados e validados pelo Intituto Nacional de Meteorologia [11] de todos os dados de entrada (isto é, Tn, Tm) para o modelo analisado, pelo período de 1961 a 1990; e

(3) Cidades com dados de umidade relativa registrados por estações meteorológicas no período de 1961 a 1990 para comparação com os valores de umidade relativa estimado pelo modelo [11].

É importante ressaltar que cidades localizadas em ambientes marinhos, porém pertencentes à região Norte do Brasil, foram excluídos das análises devido à significativa influência de rios presentes nos arredores, tornando essas cidades com características muito distintas do objetivo proposto por este artigo.

Conforme os critérios estabelecidos, foram selecionadas 16 cidades, apresentadas na Tabela 1.

Uma vez estabelecidas as cidades que atendem aos critérios adotados no presente artigo, foram realizadas análises comparativas entre os resultados dos modelos e os valores de umidade relativa efetivamente registrados por estações climatológicas pertencentes ao banco de dados fornecidos pelo Instituto Nacional de Meteorologia [11]. Para tal, foi avaliado o desempenho estatístico do modelo selecionado.

Para os testes estatísticos do modelo, foram adotados os seguintes índices estatísticos recomendados e descritos por Willmott et al. [12] e Jacovides e 
Kontoyiannis [13]: (1) o coeficiente de correlação (r); e (2) o índice de concordância (d), conforme apresentados nas Equações 4 e 5.

Tabela 1: Cidades brasileiras selecionadas para verificação dos modelos de estimativa da umidade relativa

\begin{tabular}{ccc}
\hline Referência & Cidade & Estado \\
\hline 1 & Angra dos Reis & Rio de Janeiro (RJ) \\
2 & Aracaju & Sergipe (SE) \\
3 & Cabo Frio & Rio de Janeiro (RJ) \\
4 & Caravelas & Bahia (BA) \\
5 & Florianópolis & Santa Catarina (SC) \\
6 & Ilha Guaíba & Rio de Janeiro (RJ) \\
7 & João Pessoa & Paraíba (PB) \\
8 & Macau & Rio Grande do Norte (RN) \\
9 & Maceió & Alagoas (AL) \\
10 & Natal & Rio Grande do Norte (RN) \\
11 & Recife & Pernambuco (PE) \\
12 & Rio de Janeiro & Rio de Janeiro (RJ) \\
13 & Rio Grande & Rio Grande do Sul (RS) \\
14 & Salvador & Bahia (BA) \\
15 & Santos & São Paulo (SP) \\
16 & Vitória & Espírito Santo (ES)
\end{tabular}

$$
\begin{aligned}
& r=\frac{\left[\sum_{i=1}^{n} P_{i}\left(O_{i}-\overline{O_{l}}\right)\right]}{\left[\sum_{i=1}^{n}\left(O_{i}-\overline{O_{l}}\right)^{2} \sum_{i=1}^{n}\left(P_{i}-\bar{P}_{l}\right)^{2}\right]^{0,5}} \\
& d=1-\left[\frac{\sum_{i=1}^{n}\left(P_{i}-O_{i}\right)^{2}}{\sum_{i=1}^{n}\left(\left|P_{i}-\overline{O_{l}}\right|+\left|O_{i}-\overline{O_{l}}\right|\right)^{2}}\right]
\end{aligned}
$$

Onde: $P_{i}=$ valor estimado pelo modelo; $O_{i}=$ valor observado; $\bar{P}_{l}=$ média dos valores estimados pelo modelo; $\quad \bar{O}_{l}=$ média dos valores observados; e $\mathrm{n}=$ número de dados.

\section{Resultado e Discussões}

A Figura 1 apresenta os valores de umidade relativa média observados por estações meteorológicas e estimados pelo modelo de Castellví et al. [5], no período de 30 anos (isto é, 1961 a 1990), para as cidades da costa brasileira estudadas no presente artigo (Tabela 1, apresentada previamente). 
MEDEIROS-JUNIOR, R. A.; MÜLLER, R. O., APLICAÇÃO DE MODELO PARA ESTIMAR A UMIDADE RELATIVA EM AMBIENTE MARINHO. $3^{\circ}$ Simpósio Paranaense de Patologia das Construções (30 SPPC), artigo 3SPPC1015, pp. 166 - 173, 2018. DOI:

Analisando a Figura 1, verifica-se que a maior divergência entre o valor observado e estimado para o modelo de Castellví et al. [5] foi encontrada para a cidade de Vitória, com uma diferença percentual de $9,3 \%$ entre os valores. Ou seja, a diferença dos valores não ultrapassou $10 \%$.

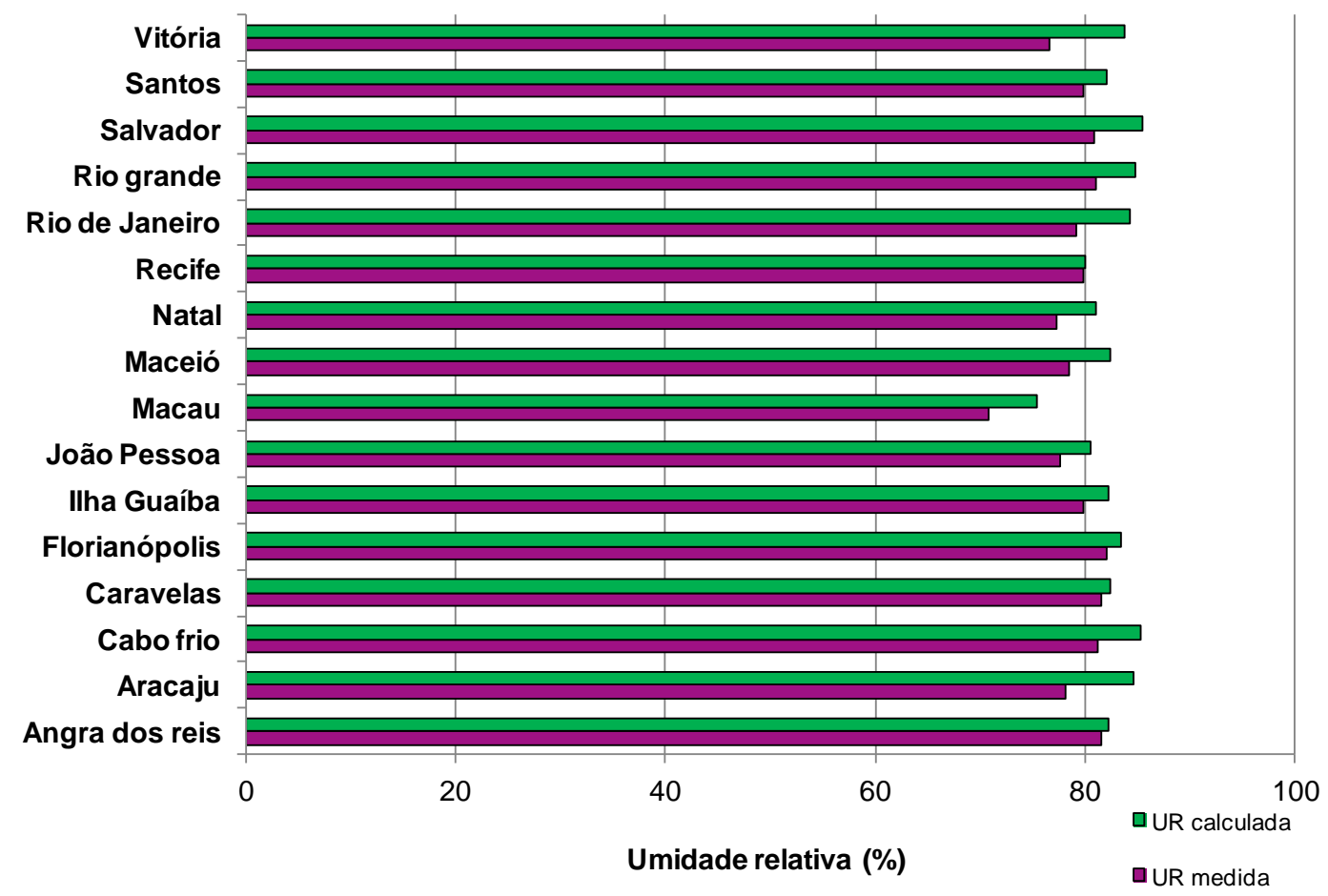

Figura 1: Valores de umidade relativa média observada por estações meteorológicas e calculada por modelo

Ainda de acordo com a Figura 1, o modelo de Castellví et al. [5] superestimou os valores de umidade relativa para todas as cidades estudadas. Os valores mais próximos encontrados foram para as cidades de Recife e Angra dos Reis, com uma diferença percentual de apenas 0,3\% e 0,7\%, respectivamente. A Figura 2 mostra a diferença percentual entre os valores medidos e calculados.

Foi avaliado o desempenho estatístico do modelo estudado e os resultados são apresentados na Figura 3. O coeficiente de correlação ( $r$ ) e 0 índice de concordância (d), calculados conforme Eqs. 4 e 5 foram 0,71 e 0,62, respectivamente.

O coeficiente $r$ é um indicativo da precisão de um modelo e revela a adequação das variáveis independentes selecionadas em explicar a variabilidade da umidade relativa ao longo do ano e entre estações meteorológicas. A correlação entre os valores é mais elevada à medida que $r$ se aproxima de 1 [12]. De acordo com a Figura 3, o coeficiente de correlação determinado para o modelo de Castellví et al. [5] relativamente próximo a $1(r=0,71)$.

Já o índice d sugere o grau de exatidão entre os valores observados e estimados por modelos, e assim como $r$, a exatidão do modelo de predição é mais elevada à medida que d se aproxima de 1 [12]. Analisando a Figura 3, observa-se que 0 
modelo de Castellví et al. [5] apresentou valor de d acima de 0,6, portanto, com alguma exatidão nas estimativas.

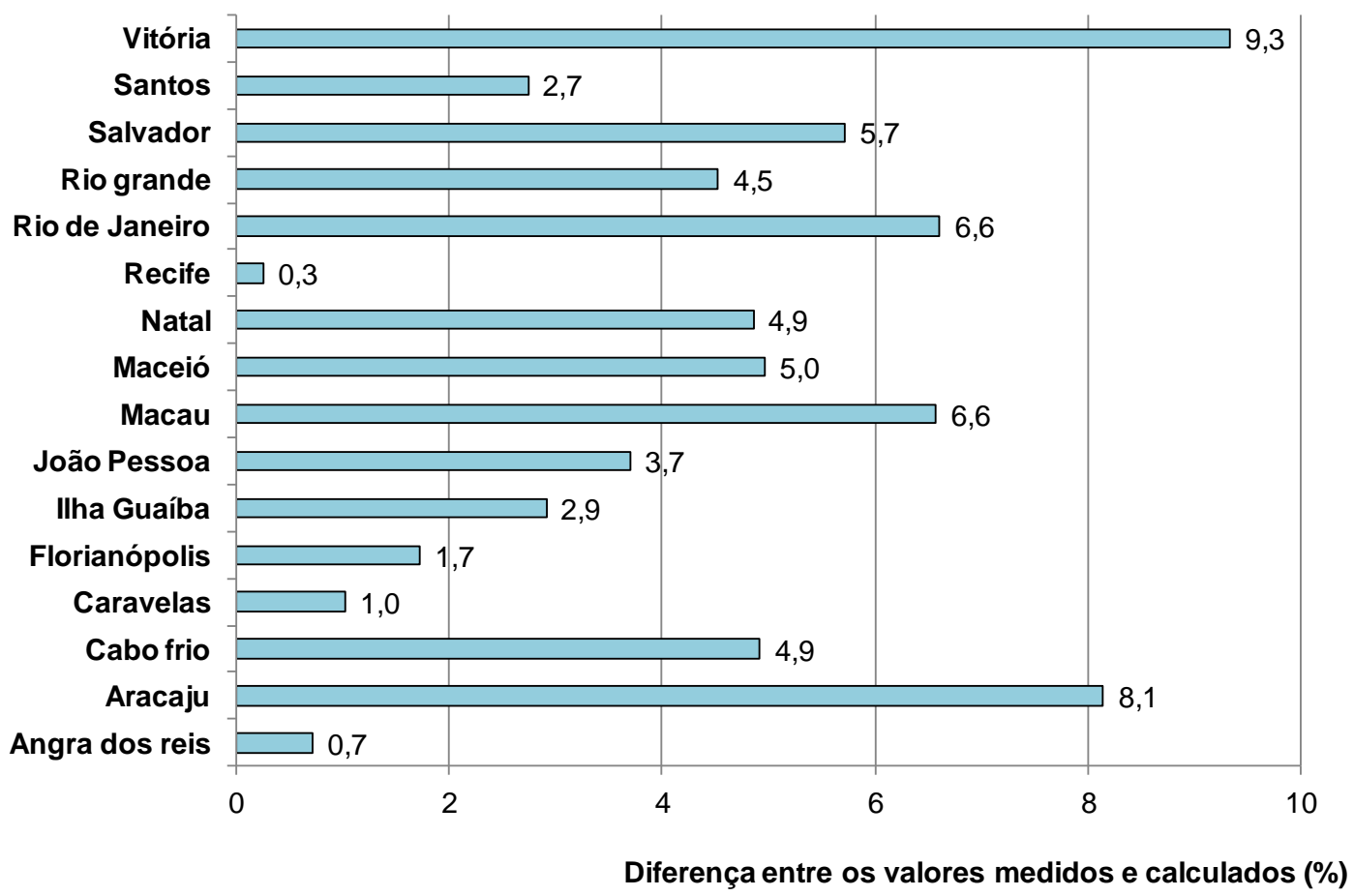

Figura 2: Variação percentual entre os valores medidos e calculados

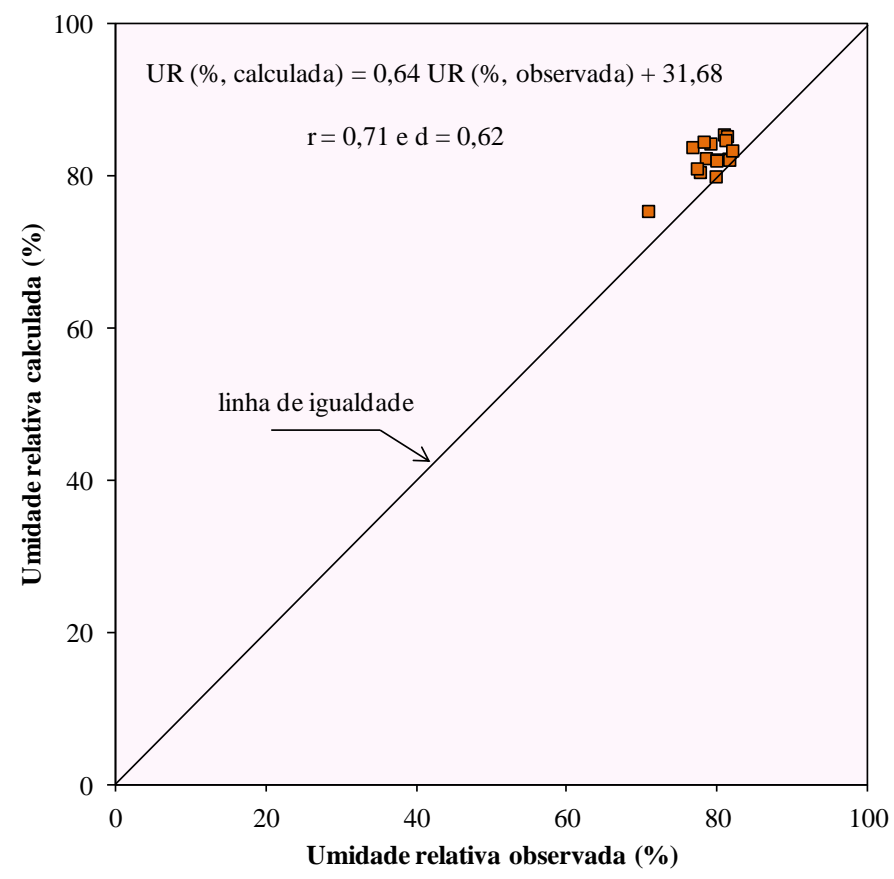

FIG. 3: Relação entre os valores observados e estimados da umidade relativa modelo de [5]

Mediante as verificações estatísticas realizadas, observa-se que o modelo de Castellví et al. [5] apresentou boas condições em estimar a umidade relativa para o 
MEDEIROS-JUNIOR, R. A.; MÜLLER, R. O., APLICAÇÃO DE MODELO PARA ESTIMAR A UMIDADE RELATIVA EM AMBIENTE MARINHO. $3^{\circ}$ Simpósio Paranaense de Patologia das Construções (30 SPPC), artigo 3SPPC1015, pp. 166 - 173, 2018. DOI: $10.4322 / 2526-7248.015$

ambiente marinho. Contudo, sugere-se o desenvolvimento ou ajuste de um modelo mais adequado para o ambiente marinho, principalmente para aplicação da umidade relativa em estudos que exijam maior precisão nos valores.

\section{Conclusões}

A umidade relativa é uma variável de extrema importância para diferentes aplicações na durabilidade e vida útil de estruturas de concreto, inclusive em se tratando de ambiente marinho.

Através de análises estatísticas, representadas pelo coeficiente de correlação $(r)$ e o índice de concordância (d), o modelo de estimativa da umidade relativa proposto por Castellví et al. [5] apresentou condições razoáveis ( $r=0,71$ e $d=0,62)$ para estimar a umidade relativa em ambientes marinhos, revelando-se uma ferramenta útil para preenchimento de séries de dados.

Ressalta-se, no entanto, que a sua aplicabilidade pode ser comprometida para estudos que necessitem maior exatidão na estimativa dessa variável para 0 ambiente marinho. Recomenda-se a continuidade da validação do modelo para outras regiões, para confirmar a tendência de exatidão do modelo.

\section{Agradecimentos}

Os autores gostariam de agradecer a Universidade Federal do Paraná (UFPR) e ao Programa de Pós-Graduação em Engenharia de Construção Civil (PPGECC).

\section{Referências}

[1] Mazer, W.; Lima, M.G.; Medeiros-Junior, R.A. (2017) Fuzzy logic for estimating the chloride diffusion in concrete, Proceedings of ICE - Structures and Buildings, 170: http://dx.doi.org/10.1680/jstbu.16.00153

[2] Medeiros-Junior, R.A.; Lima, M.G.; Brito, P.C., Medeiros, M.H.F. (2015) Chloride penetration into concrete in an offshore platform analysis of exposure conditions, Ocean Engineering, 103:78-87.

[3] Bastidas-Arteaga, E.; Chateauneuf, A.; Sánchez-Silva, M.; Bressolette, Ph.; Schoefs, F. (2010) Influence of weather and global warming in chloride ingress into concrete: a stochastic approach, Structural Safety, 32:238-249.

[4] Tardivo, G. ; Berti, A. (2012) A dynamic method for gap filling in daily temperature datasets, Journal of Applied Meteorology and Climatology, 51:1079-1086.

[5] Castellví, F.; Perez, P.J.; Villar, J.M.; Rosell, J.L. (1996) Analysis of methods for estimating vapor pressure deficits and relative humidity, Agricultural and Forest Meteorology, 82:29-45.

[6] Tetens, O. (1930) Über einige meteorologische Begriffe, Zeitschrift für Geophysik, 6:297-309.

[7] Kuo, H.L.; Raymond, W.H. (1980) A quasi-one-dimensional cumulus cloud model and parameterization of cumulus heating and mixing effects, Monthly Weather Review, 108:991-1009. 
[8] Raymond, W.H. (2000) Moisture advection using relative humidity, Journal of Applied Meteorology, 39:2397-2408.

[9] Vuille, M.; Bradley, R.S.; Werner, M.; Keimig, F. (2003) 20TH century climate change in the Tropical Andes: observations and model results, Climatic Change, 59:75-99.

[10] Andrade, C.; Castillo, A. (2003) Evolution of reinforcement corrosion due to climatic variations, Materials and Corrosion, 54:379-386.

[11] INMET (1992) National Institute of Meteorology, in Climatological normals 1961 1990, A. M. Ramos, L. A. R. Santos, and L. T. G. Fortes, editors, CD-ROM, Brazil, pp. 1-465.

[12] Willmott, C.J.; Ackleson, S.G.; Davis, R.E.; Feddema, J.J.; Klink, K.M.; Legates, D.R.; O'Donnell, J.; Rowe, C.M. (1985) Statistics for the evaluation and comparison of models, Journal of Geophysical Research, 90: 8995-9005.

[13] Jacovides, C.P.; Kontoyiannis, H. (1995) Statistical procedures for the evaluation of evapotranspiration computing models, Agricultural Water Management, 27:365-371. 